\title{
ON-OFF INTERMITTENCY IN RANDOMLY DRIVEN NONLINEAR FERROMAGNETIC RESONANCE
}

\author{
A. Krawiecki and A. Sukiennicki \\ Institute of Physics, Warsaw University of Technology \\ Koszykowa 75, 00-662 Warszawa, Poland
}

(Received October 19, 1995; in final form December 1, 1995)

\begin{abstract}
Simple models of nonlinear ferromagnetic resonance are considered which describe perpendicular resonance and parallel pumping with the rf field amplitude changing randomly and chaotically in time. On-off intermittency is obtained from the numerical solution of the equations of motion for the spin-wave amplitudes when the mean value of the rf field amplitude exceeds the Suhl instability threshold. Possible experimental applications are discussed.
\end{abstract}

PACS numbers: $05.45+\mathrm{b}, 76.50 .+\mathrm{g}, 75.30 . \mathrm{Ds}$

\section{Introduction}

On-off intermittency (OOI) [1-6] occurs in dynamic systems which possess a quasi-invariant set within their phase space, i.e. a state near to which the phase trajectory tends to spend long stretches of time. $\mathrm{OOI}$ is characterized by a sequence of flat (laminar) phases, when the observed signal is close to zero, and bursts, when the signal assumes non-zero values and behaves chaotically. The bursts occur when the control parameter $\varepsilon$ exceeds some critical value $\varepsilon_{\mathrm{c}}$. The appearance of bursts is connected with the loss of stability of the invariant set which results from dynamic time-dependent forcing of the bifurcation parameter (which is related, but not necessarily identical to the control parameter) through the bifurcation point. These characteristics distinguish OOI from other types of intermittency, for which the parameters are static, such as Pomeau-Maneville intermittency types I-III [7] or crisis-induced intermittency [8], which consist in switching the system between a periodic and chaotic state or between two different chaotic states, respectively.

For many systems it is possible to destabilize the invariant set externally, by replacement of the system control parameter with a time-dependent variable. If this variable is stochastic, the system is referred to as randomly (or stochastically) driven, while when the control parameter varies in time chaotically, in the sense 
of deterministic chaos, the system is referred to as chaotically driven. Following Refs. [1-3] we distinguish here between these two situations. Formally, this discrimination is justified because in the case of chaotic drive the description of the system is purely deterministic, but in the case of random drive one must appeal to probabilistic methods.

So far, OOI has been reported to occur in a randomly or chaotically driven logistic map [2,3] (see below), coupled map lattices [9], in a system of coupled ordinary differential equations which in particular limit reduces to the Lorenz system [1], in a simple model of chaos in nonlinear ferromagnetic resonance [10], and observed experimentally in nonlinear RLC circuits [11-13] and chaotic nonlinear ferromagnetic resonance [14]. It seems interesting to find other physical systems exhibiting this kind of intermittency, in particular such in which the experiments may be performed. In this paper we provide a numerical evidence that OOI may be observed in high-power ferromagnetic resonance in perpendicular and parallel pumping, just above the Suhl instability threshold, if the control parameter - the rf field amplitude - is varied randomly or chaotically in time, slowly in comparison with the rf field frequency.

The essential features of OOI are easiest to describe by means of a randomly driven logistic map [2,3], defined as $y_{n+1}=\varepsilon_{n} y_{n}\left(1-y_{n}\right)+\xi_{n}$. Here, $\varepsilon_{n}=\varepsilon x_{1, n}$, $\xi_{n}=\xi x_{2, n}$, and $x_{1, n}, x_{2, n}$ are random uncorrelated variables in the interval $[0,1]$ with uniform distribution; $\varepsilon>1$ is the control parameter, $\varepsilon_{n}$ is the time-dependent bifurcation parameter and the last term represents white noise with the amplitude $\xi$. If $\varepsilon>\varepsilon_{\mathrm{c}}$ and $\xi=0, y_{n}$ no longer converges to the fixed point $\widehat{y}=0$ (the invariant set for this map), but bursts in a chaotic manner, and the bursts are separated by laminar phases for which $y_{n} \approx 0$. For given $\varepsilon$ the probability that a laminar phase has the length $\tau$ is $P(\tau)=A \exp \left(-B \tau \delta^{2}\right) \tau^{-3 / 2}$, where $\delta=\left|\varepsilon-\varepsilon_{\mathrm{c}}\right|$, or $P(\tau) \propto \tau^{-3 / 2}$ for sufficiently small $\tau, \delta$. The mean laminar phase length changes with the control parameter as $\langle\tau\rangle=\alpha+\beta \delta^{-1}(A, B, \alpha, \beta$ are constants). If $\xi>0$, the $\tau^{-3 / 2}$ law for the laminar phase lengths distribution is valid only for small $\tau$ and the exponential decay of $P(\tau)$ begins for $\tau \simeq \tau^{\star}$ substantially smaller than in the noise-free case. The crossover time $\tau^{\star}$ scales with the noise amplitude as $\tau^{\star} \propto\left[\ln \left(y_{\mathrm{t}} / \xi\right)\right]^{2}$, where $y_{\mathrm{t}}$ is the "onset threshold" for a burst, which may be chosen quite freely.

These characteristics were derived analytically for the above-mentioned map only, but there exists strong evidence that they remain valid for a large class of systems exhibiting OOI, e.g. for a chaotically driven logistic map for which the bifurcation parameter varies in time chaotically, instead of randomly, and for time-continuous systems [2,9-14]. An intriguing property of OOI is that these characteristics seem to be insensitive to the discrimination between random and chaotic drive [2].

\section{The model}

High-power ferromagnetic resonance (for review of the theory see e.g. [15]) has been for almost fifteen years a useful tool in the study of nonlinear phenomena, including, e.g. multistability [16], auto-oscillations of the magnetization and chaos [16-22], hyperchaos [23], control of chaos [24,25], various types of intermittency $[26-28]$, chaotic transients $[29,30]$ etc. Less attention was devoted to the case 
in which the rf field amplitude varies stochastically in time, e.g. stationary state above the parallel pumping instability threshold was investigated in Ref. [31]. In this paper we deal with two resonance configurations, namely, with the perpendicular resonance in coincidence regime, when the uniform mode is driven in resonance, and with the parallel pumping. We consider a case when the rf field amplitude varies randomly or chaotically in time slowly in comparison with the pumping frequency.

Let us assume first that the rf field amplitude is constant. As it exceeds the Suhl instability threshold, in the case of perpendicular resonance the uniform mode, excited directly by the rf field, decays into a pair of spin waves (SW) with half the pumping frequency and opposite wave vectors [32]. In the case of parallel pumping the rf field excites directly a pair of SW with half the pumping frequency and opposite wave vectors [33]. These phenomena are called the first-order Suhl instability and parallel pumping instability, respectively.

Henceforth we assume that the rf field time dependence in both cases is $h(t)=\tilde{h}(t) \cos \omega t$, where $\omega$ is the pumping frequency on the order of some $\mathrm{GHz}$, and $\tilde{h}(t)$ is the time-dependent rf field amplitude which varies in time randomly or chaotically, slowly in comparison with $\cos \omega t$. Always when $\tilde{h}(t)$ increases above the Suhl threshold, the instability occurs. We assume that only one pair of SW is excited via the decay of the uniform mode or by the rf field, depending on the instability type (see Sec. 4. for discussion). The uniform mode will be labelled by zero, the parametric SW pair by $k,-k$ and their complex amplitudes by $a_{0}, a_{k}$, $a_{-k}$. The canonical equations of motion for the mode amplitudes may be obtained from the magnetic Ilamiltonian written in terms of SW creation and annihilation operators [15], as in the case of constant in time of field amplitude $\tilde{h}$ with the change $\tilde{h} \rightarrow \tilde{h}(t)$. The Hamiltonian for the perpendicular resonance case contains only an interaction term between the rf field and the uniform mode as well as a three-mode interaction term between the uniform mode and the parametric SW pair. In the case of parallel pumping, apart from a coupling term between the rf field and the SW pair, it is necessary to include a four-mode interaction term which assures the existence of a fixed point of the equations of motion above the instability threshold. As $\tilde{h}(t)$ varies slowly in time, it is possible to rewrite the equations in rescaled variables $u_{0}=\left|V_{0, k}\right| \exp (-\mathrm{i} \omega t) a_{0}, u_{k}=\left|V_{0, k}\right| \exp (-\mathrm{i} \omega t / 2) a_{k}$ in the case of perpendicular resonance and $u_{k}=\left(S_{k, k}+2 T_{k, k}\right)^{1 / 2} \exp (-\mathrm{i} \omega t / 2) a_{k}$ in the case of parallel pumping. The variables $u_{0, k}$ also vary slowly in time, because the fast time dependence is cancelled by the exponential term. It is also possible to assume $u_{k}=u_{-k}[15,31]$. In the above definitions $V_{0, k}$ is the three-mode coupling coefficient between the uniform mode and the SW pair and $S_{k, k}, T_{k, k}$ are four-mode coupling coefficients in the $S$-theory of parallel pumping (for definitions see e.g. [19]).

The final form of the equations of motion in the variables $u_{0}, u_{k}$ for the perpendicular resonance is

$$
\begin{aligned}
& \dot{u}_{0}=-\mathrm{i} \varepsilon(l) \eta_{0} \eta_{k}-\eta_{0} u_{0}-\mathrm{i} u_{k}^{2}+\xi_{0}, \\
& \dot{u}_{k}=-\eta_{k} u_{k}-\mathrm{i} u_{0} u_{k}^{\star}+\xi_{k}
\end{aligned}
$$


and for the parallel pumping

$$
\dot{u}_{k}=-\eta_{k} u_{k}-\mathrm{i} \eta_{k} \varepsilon(t) u_{k}^{\star}-\mathrm{i}\left|u_{k}\right|^{2} u_{k}+\xi_{k} .
$$

Besides the terms coming from the Hamiltonian, phenomenological SW damping $\eta_{0, k}$ and thermal noise $\xi_{0, k}$ were added in the above equations as parameters. By including the latter term one accounts for the fact that even with the rf field turned off SW are thermally excited to some equilibrium level $u_{k \text {,therm }} \simeq \xi_{k} / \eta_{k}$. The time-dependent bifurcation parameter is $\varepsilon(t)=\tilde{h}(t) / h_{\mathrm{thr}}$, where $h_{\mathrm{thr}}$ is the instability threshold. Henceforth we define $\varepsilon(t)=\varepsilon x(t)$. Now $\varepsilon$ is the control parameter and $x(t)$ varies slowly in time, randomly or chaotically, in the interval $[0,1]$. For the rf field amplitude constant in time in both above equations the Suhl instability threshold is $\varepsilon=1$.

The parameter values in Eqs. $(1,2)$ may be estimated as follows. The SW damping $\eta_{0}, \eta_{k}=1.0 \times 10^{6} \mathrm{~s}^{-1}$ was assumed. $\xi_{k}$ may be estimated from the equalities $\xi_{k} / \eta_{k}=u_{k, \text { therm }}=\left|V_{0, k} a_{k, \text { therm }}\right|=\left|V_{0, k}\right|\left(\hbar \bar{n}_{k}\right)^{1 / 2}$ and $\xi_{k} / \eta_{k}=\left(S_{k, k}+\right.$ $\left.2 T_{k, k}\right)^{1 / 2}\left(\hbar \bar{n}_{k}\right)^{1 / 2}$ in the case of perpendicular resonance and parallel pumping, respectively, where $\bar{n}_{k}$ is the a verage number of magnons with energy $\hbar \omega_{k}=\hbar \omega / 2$ in the given temperature, resulting from the Bose-Einstein distribution. The values of the coupling coefficients depend on the wave vector and propagation direction of the parametric SW pairs; they are on the order of $V_{0, k} \simeq 4 \pi \gamma M_{0}\left(2 \gamma / M_{0}\right)^{1 / 2}$, $S_{k, k}, T_{k, k} \simeq 2 \pi \gamma^{2}$ [15], where $\gamma=1.77 \times 10^{7} \mathrm{G}^{-1} \mathrm{~s}^{-1}$ is the gyromagnetic ratio and $M_{0}$ is the saturation magnetization ( $=1750$ Oe for yttrium iron garnet YIG). Thus, $u_{k \text {,therm }} \simeq 10^{-4}-10^{-3} \mathrm{~s}^{-1}$ in the case of perpendicular resonance and $10^{-9}-10^{-8} \mathrm{~s}^{-1 / 2}$ in the case of parallel pumping, for the temperature ranging from liquid helium to room temperature. (Let us note that the variables $u_{0, k}$ in Eq. (1) have the dimension $\mathrm{s}^{-1}$ and in Eq. (2) $\mathrm{s}^{-1 / 2}$.) In Eq. (1), $\xi_{0}=\xi_{k}$ is assumed for simplicity.

It is useful to divide both sides of Eqs. $(1,2)$ by $\eta_{k}$ and work with renormalized time $\eta_{k} t$. The amplitudes $u_{0, k}$ were divided by $10^{6}$ in Eq. (1) and by $10^{3}$ in Eq. (2), so that their maximum values were on the order of unity. We integrated the equations numerically for two different realizations of the random and chaotic time dependence of the control parameter $\varepsilon(t)$. Namely, in a case of randomly driven ferromagnetic resonance (case $A$ ) $x(t)$ was assumed to be a random square wave, i.e. a function changing its value randomly in the interval $[0,1]$ every $\Delta t$ renormalized time units. In a case of chaotically driven ferromagnetic resonance (case B) $x(t)=(0.5 / 27) \times\left(x_{2}(t)+27\right)$ was a rescaled variable from a well-known chaotic Lorenz system [34]

$$
\begin{aligned}
& \dot{x_{1}}=-s \sigma\left(x_{1}-x_{2}\right), \\
& \dot{x_{2}}=s\left(-x_{1} x_{3}+r x_{1}-x_{2}\right), \\
& \dot{x_{3}}=s\left(x_{1} x_{2}-b x_{3}\right) .
\end{aligned}
$$

Time units in Eqs. (3) are renormalized time units of Eqs. $(1,2)$, but the factor $s$ changes the characteristic time scale; $r=28, \sigma=10, b=8 / 3$ and $x(t)$ is defined so that its values are contained in the $[0,1]$ interval and its mean value $\langle x(t)\rangle_{t}=0.5$. In case (A), like in the experiment of Ref. [11], Eqs. $(1,2)$ are 
turned into a discrete-time map, but such a random square wave may serve as an approximation for a stochastic signal varying in time with the characteristic time scale $\Delta t$. The integration scheme was a fourth-and-fifth order Runge-Kutta method with permanent step size and error control.

\section{Results}

If $\varepsilon<2$, in both cases (A) and (B) the SW amplitudes $u_{k}$ in Eqs. $(1,2)$ decrease to zero; in Eq. (1) only the uniform mode amplitude $u_{0}$ assumes non-zero values. If $\varepsilon>\varepsilon_{\mathrm{c}}=2$, the mean value of the normalized rf field amplitude $\varepsilon(t)$ exceeds the Suhl instability threshold 1.0 and bursts of the SW amplitudes occur. These bursts (Fig. 1) exhibit the characteristics of OOI. In order to observe the bursts in a sufficiently wide region of the control parameter, the characteristic time scale of the oscillations of the function $x(t)$ must be comparable or greater than the reverse of the phenomenological damping $\eta_{k}$. Thus we assumed $\Delta t=2.0$ in case (A) and $s=1 / 20$ in Eqs. (3) in case (B). We set the onset threshold for the burst $u_{k, t}$ between 0.2 and $3.91 \times 10^{-4}$ a.u., but the results turned out to be quite insensitive to these values.

The results are summarized in Figs. 2-5. Figures 2 and 3 were obtained without thermal noise $\left(\xi_{k}=10^{-300}\right.$ was set in order to avoid the convergence of the SW amplitudes to numerical zero during long laminar phases). From Fig. 2a one can see that the probability distribution for the laminar phase lengths in all cases scales as $\tau^{-3 / 2}$ for sufficiently small $\tau$. However, e.g. for the case of the perpendicular pumping driven by the Lorenz signal (case (B), Eq. (1)) exponential decay of this distribution is observed for longer laminar phases, too (Fig. 2b). The mean laminar phase length scales as $\delta^{-1}$ (Fig. 3).

In Fig. 4 the effect of thermal noise $\xi_{k} \neq 0$ on $P(\tau)$ is shown. The probability distribution exhibits a characteristic shoulder, as in Ref. [3], and decays exponentially already for small $\tau$. The characteristic crossover time $\tau^{\star}-$ as defined in Sec. 1 - may be found by determining, for each $\xi_{k}$, the point at which the extrapolated $-3 / 2$ power law line crosses the trailing edge of the shoulder [11]; such points are signed by arrows in Fig. 4. In Fig. 5 the dependence of $\tau^{\star}$ on the thermal noise
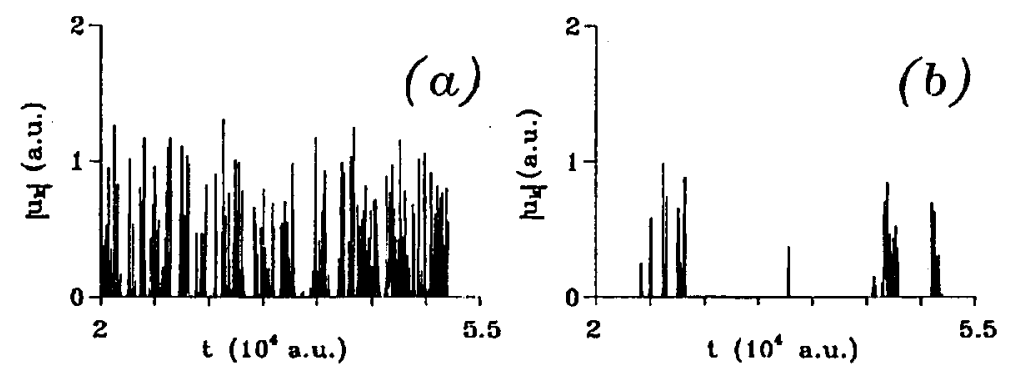

Fig. 1. Time series for the parametric spin wave amplitude $\left|u_{k}\right|$ for randomly driven perpendicular resonance: (a) $\varepsilon=2.1$, (b) $\varepsilon=2.01$. 

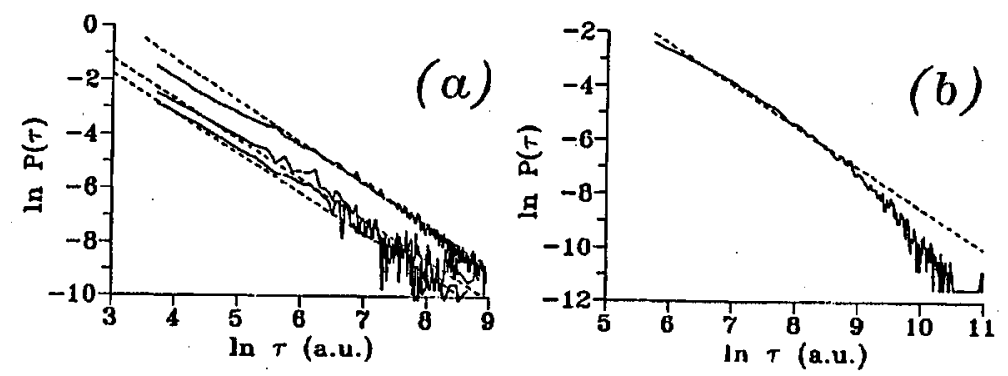

Fig. 2. (a) Probability distribution $P(\tau)$ for randomly driven parallel pumping (bottom curve) and for randomly (middle) and chaotically (top) driven perpendicular resonance; dotted lines have the slope $-3 / 2, \varepsilon=2.033$. (b) As in (a), but with an extended time scale, for chaotically driven perpendicular resonance. The sample was $10^{5}$ laminar phases.
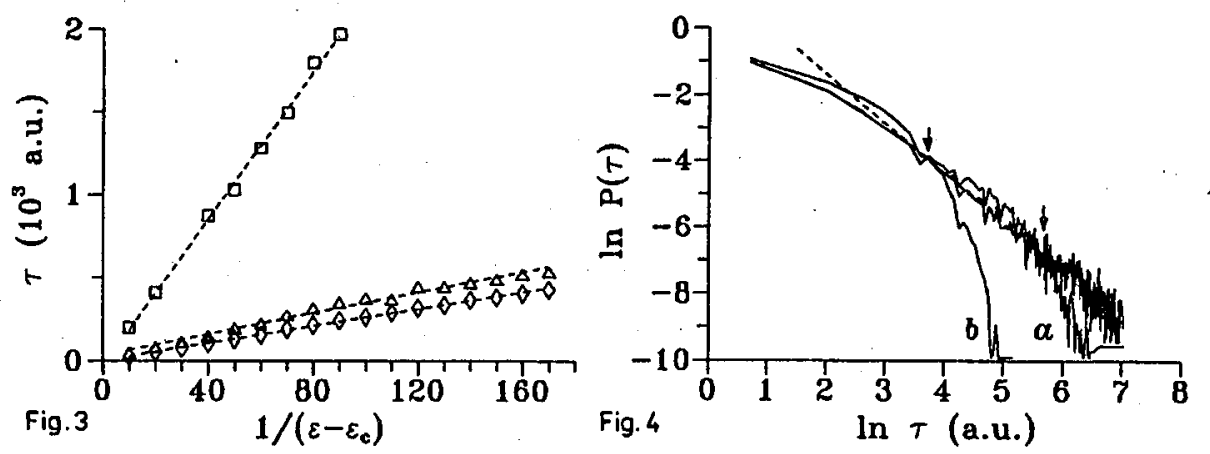

Fig. 3. Mean length of the laminar phase for randomly driven parallel pumping (diamonds) and for randomly (triangles) and chaotically (squares) driven perpendicular resonance. Every point was obtained as an average over $2 \times 10^{4}$ laminar phases.

Fig. 4 Effect of thermal noise on the probability distribution $P(\tau)$ for randomly driven perpendicular resonance; $\varepsilon=2.033, u_{k, t}=3.91 \times 10^{-4}$, curve (a) is for $\xi_{k} / \eta_{k}=0.0195$, curve (b) for $\xi_{k} / \eta_{k}=10$.

amplitude is shown. One can see that the scaling law $\tau^{\star} \propto\left[\ln \left(u_{k, t} / \xi_{k}\right)\right]^{2}$ is fulfilled quite well. We kept the onset threshold for the burst constant, $u_{k, t}=3.91 \times 10^{-4}$ in both cases (A), (B), and varied $\xi_{k} / \eta_{k}$ between 10 and $1.5 \times 10^{-4}$ so that to obtain a sufficiently wide scaling area. Such values of $\xi_{k} / \eta_{k}$ are some orders of magnitude greater than the estimates from Sec. 2, however, for small thermal noise amplitudes the uncertainties of $\tau^{\star}$, estimated as in Fig. 5, substantially increase. A similar scaling law might be obtained experimentally by varying $u_{k, t}$ with the given thermal noise level.

We obtained numerically OOI for other chaotic and random drives, too. In all cases, the numerical results agreed well with the qualitative characteristics of OOI, again proving their universality. The differences between the systems with different drive types (e.g. random and chaotic or various chaotic drives) are only 

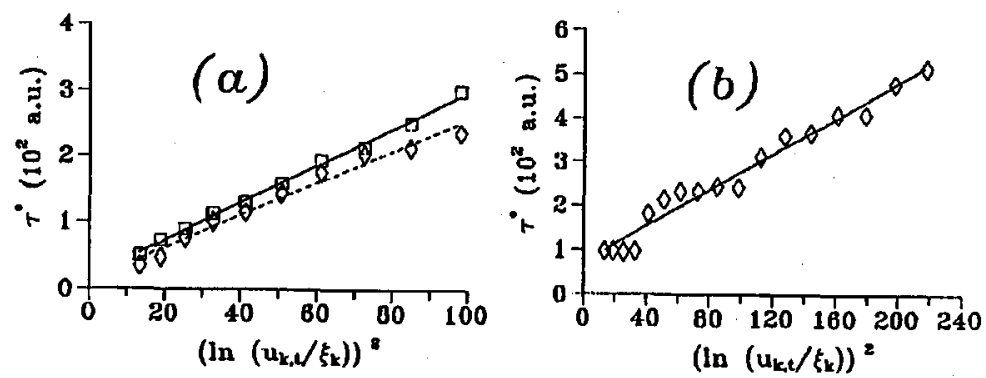

Fig. 5. (a) The scaling of the crossover time $\tau^{\star}$ for randomly driven parallel pumping (diamonds) and perpendicular resonance (squares); $u_{k, t}=3.91 \times 10^{-4}$. (b) As in (a), for chaotically driven perpendicular resonance.

quantitative, as here between cases A and B. This is, of course, caused by different drive characteristics, but this problem has not been investigated in detail so far.

\section{Discussion and conclusions}

We have shown that in simple models of high-power perpendicular resonance and parallel pumping it is possible to obtain $\mathrm{OOI}$ in the time series of the amplitudes of parametric SW pairs. For this purpose the rf field amplitude should vary randomly or chaotically in time with the frequency on the order of hundreds of $\mathrm{kHz}$, comparable or smaller than the SW damping rate, and its mean value should slightly exceed the first-order or parallel pumping instability threshold. The reason for the appearance of OOI is that at the instability threshold the stable fixed points of Eqs. $(1,2)$ with $u_{k}=0$ loose stability, and new stable fixed points appear with non-zero SW amplitudes dependent on the rf field amplitude. This is the same scenario as in the randomly driven logistic map in which for $\varepsilon>1$ the fixed point $\widehat{y}=0$ is unstable and another fixed point $\widehat{y}(\varepsilon)$ exists. The presented results for our time-continuous system are in good agreement with the theory of OOI developed for discrete-time maps.

The experimental realization seems possible. In the parallel pumping case it is sufficient to observe the absorption, which is proportional to the amplitudes of parametric SW, with the sampling frequency equal to some MHz. In the perpendicular resonance case one could measure electromagnetic radiation with frequency $\omega / 2$ from the sample in the shape of a thin film, like in the experiment in Ref. [35].

A question arises if such simple models as Eqs. $(1,2)$, only with one pair of parametric SW, can describe the experimental situation. However, for the case of parallel pumping and constant in time rf field amplitude it may be shown that (slightly simplifying the problem) only one SW pair is excited just above the instability threshold [15], while the excitation of other SW pairs requires significantly increased pumping power; similar results were obtained experimentally for the perpendicular resonance [36]. As in our equations of motion OOI is observed when $\langle\tilde{h}(t)\rangle_{t}$ exceeds only a little the instability threshold, it is reasonable to assume that only one pair of SW is excited. On the other hand, the statistics of the laminar phases may be influenced by four-mode interactions of the excited SW pair with 
other, thermally excited magnons. An effect of such interactions on the occurrence of the stationary state, with one pair of SW excited well above the thermal level from the uniform distribution of thermally excited magnons, was considered, e.g. in Refs. [37,38] (see also [15]). Similar problems may be found when considering the appearance of bursts in our case. Besides, nothing is known about OOI in such high-dimensional systems. Thus more experimental and theoretical work is needed to answer the above-mentioned question.

The results of the present paper together with Refs. $[10,14]$ indicate that the high-power ferromagnetic resonance is a promising method for the investigation of OOI in magnetic systems and support the conjecture that this kind of intermittency is present in a variety of nonlinear systems [11].

\section{References}

[1] N. Platt, E.A. Spiegel, C. Tresser, Phys. Rev. Lett. 70, 279 (1993).

[2] J.F. Heagy, N. Platt, S.M. Hammel, Phys. Rev. E 49, 1140 (1994).

[3] N. Platt, S.M. Hammell, J.F. Heagy, Phys. Rev. Lett. 72, 3498 (1994).

[4] A.S. Pikovsky, Z. Phys. B, Condens. Matter 55, 149 (1984).

[5] H. Fujisaka, H. Yamada, Progr. Theor. Phys. 74, 918 (1985); 75, 1087 (1986).

[6] E. Ott, J.C. Sommerer, Phys. Lett. A 188, 39 (1994).

[7] Y. Pomeau, P. Maneville, Commun. Math. Phys. 74, 1889 (1980).

[8] C. Grebogi, E. Ott, F. Romeiras, J.A. Yorke, Phys. Rev. A 36, 5365 (1987).

[9] H.L. Yang, E.J. Ding, Phys. Rev. E 50, R3295 (1994).

[10] A. Krawiecki, A. Sukiennicki, Acta Phys. Pol. A 88, 269 (1995).

[11] P.W. Hammer, N. Platt, S.M. Hammel, J.F. Heagy, B.D. Lee, Phys. Rev. Lett. 73, 1095 (1994).

[12] Y.H. Yu, K. Kwak, T.K. Lim, Phys. Lett. A 198, 34 (1995).

[13] A. Čenys, A. Namajūnas, A. Tamaševičius, private communication.

[14] F. Rödelsperger, A. Čenys, H. Benner, to appear in Phys. Rev. Lett.

[15] V. Zakharov, V. Lvov, S.S. Starobinets, Usp. Fiz. Nauk 114, 609 (1974) [Sov. Phys. - Usp. 17, 896 (1975)].

[16] G. Wiese, H. Benner, Z. Phys. B, Condens. Matter 79, 119 (1990).

[17] G. Gibson, C. Jeffries, Phys. Rev. A 29, 811 (1984).

[18] K. Nakamura, S. Ohta, K. Kawasaki, J. Phys. C, Solid State Phys. 15, L143 (1982).

[19] P.H. Bryant, C.D. Jeffries, K. Nakamura, Phys. Rev. A 38, 4223 (1988).

[20] M. Mino, S. Mitsudo, H. Yamazaki, J. Magn. Magn. Mater. 104-107, 1055 (1992).

[21] G. Srinivasan, M. Chen, C.E. Patton, J. Appl. Phys. 64, 5480 (1988).

[22] R.D. McMichael, P.E. Wigen, Phys. Rev. Lett. 64, 64 (1989).

[23] H.R. Moser, P.F. Meier, F. Waldner, Phys. Rev. B 47, 217 (1993).

[24] M. Ye, D.E. Jones, P.E. Wigen, J. Appl. Phys. 73, 6822 (1993).

[25] A. Azevedo, S.M. Rezende, Phys. Rev. Lett. 66, 1342 (1991).

[26] F.M. de Aguiar, A. Azevedo, S.M. Rezende, J. Appl. Phys. 73, 6825 (1993).

[27] F.M. de Aguiar, Phys. Rev. A 40, 7244 (1989). 
[28] F. Rödelsperger, T. Weyrauch, H. Benner, J. Magn. Magn. Mater. 104-107, 1075 (1992).

[29] F.M. de Aguiar, S.M. Rezende, F.C.S. da Silva, J. Appl. Phys. 75, 5616 (1994).

[30] T.L. Carroll, L.M. Peccora, F.J. Rachford, Phys. Rev. A 40, 377 (1989).

[31] V.B. Cherepanov, Fiz. Tverd. Tela 22, 43 (1980) [Sov. Phys. - Solid State, Jan. 1980, p. 25].

[32] H. Suhl, J. Phys. Chem. Solids 1, 209 (1957).

[33] E. Schlömann, R.I. Joseph, J. Appl. Phys. 32, 1006 (1961).

[34] E.N. Lorenz, J. Atmos. Sci. 20, 130 (1963).

[35] G.A. Melkov, S.V. Sholom, Zh. Eksp. Teor. Fiz. 96, 712 (1989) [Sov. Phys. - JETP $69,403(1989)]$.

[36] P. Kabos, G. Wiese, C.E. Patton, Phys. Rev. Lett. 72, 2093 (1994).

[37] V.E. Zakharov, V.S. Lvov, S.L. Musher, Fiz. Tverd. Tela 14, 832 (1972) [Sov. Phys. - Solid State 14, 710 (1972)].

[38] S.P. Lim, D.L. Huber, Phys. Rev. B 41, 9283 (1990). 